Draft VERSion SEPTEMBER 11, 2018

Preprint typeset using LATEX style AASTeX6 v. 1.0

\title{
INTERFERENCE AS AN ORIGIN OF THE PEAKED NOISE IN ACCRETING X-RAY BINARIES
}

\author{
Alexandra Veledina ${ }^{1,2,3}$ \\ ${ }^{1}$ Nordita, KTH Royal Institute of Technology and Stockholm University, Roslagstullsbacken 23, SE-10691 Stockholm, Sweden \\ ${ }^{2}$ Tuorla Observatory, University of Turku, Väisäläntie 20, FI-21500 Piikkiö, Finland; alexandra.veledina@gmail.com \\ ${ }^{3}$ Nordita Fellow
}

\begin{abstract}
We propose a physical model for the peaked noise in the X-ray power density spectra of accreting X-ray binaries. We interpret its appearance as an interference of two Comptonization continua: one coming from the up-scattering of seed photons from the cold thin disk and the other fed by the synchrotron emission of the hot flow. Variations of both X-ray components are caused by fluctuations in mass accretion rate, but there is a delay between them corresponding to the propagation timescale from the disk Comptonization radius to the region of synchrotron Comptonization. If the disk and synchrotron Comptonization are correlated, the humps in the power spectra are harmonically related and the dips between them appear at frequencies related as odd numbers 1:3:5. If they are anti-correlated, the humps are related as 1:3:5, but the dips are harmonically related. Similar structures are expected to be observed in accreting neutron star binaries and supermassive black holes. The delay can be easily recovered from the frequency of peaked noise and further used to constrain the combination of the viscosity parameter and disk height-to-radius ratio $\alpha(H / R)^{2}$ of the accretion flow. We model multi-peak power spectra of black hole X-ray binaries GX 339-4 and XTE J1748-288 to constrain these parameters.
\end{abstract}

Keywords: accretion, accretion disks - X-rays: binaries - black hole physics

\section{INTRODUCTION}

Astrophysical black holes can be studied by both spectral and timing techniques. Spectral approach was more used in the beginning of the X-ray era due to the typically little amount of X-ray photons. Temporal properties on short timescales, down to milliseconds, are perhaps the most challenging to probe. A revolution in the field of black hole astrophysics was made by the X-ray mission Rossi X-ray Timing Explorer, with the help of which a vast amount of information on Galactic compact objects was collected. It was realized that many properties of these objects can be understood by studying their variability patterns, which depend greatly on the spectral state and black hole mass.

The X-ray power spectral densities (PSDs) of black hole binaries generally demonstrate aperiodic variability and the low-frequency quasi-periodic oscillations (QPOs, Casella et al. 2005). Early studies suggested that the hard-state broadband PSD constitutes a double-broken power-law with a $f^{-1}$ dependence ( $f$ is the Fourier frequency) in the range $\sim 0.01-10 \mathrm{~Hz}$, below and above this range the variability is suppressed (Nolan et al. 1981; Belloni \& Hasinger 1990b; Churazov et al. 2001). This $f^{-1}$ power-law was successfully reproduced in the model of propagating fluctuations of mass accretion rate (Lyubarskii 1997; Kotov et al. 2001; Arévalo \& Uttley 2006). A model was extended by studies of fluctuations damping (Zdziarski et al. 2009) and modeling of the QPO (Ingram et al. 2009; Ingram \& Done 2011; Veledina et al. 2013a). It was also examined with global accretion disk simulations (Hogg \& Reynolds 2015). Yet all these investigations obtain an originally proposed power-law $f^{-1}$ in the frequency range of interest. The PSDs obtained in propagating fluctuations model can now be directly fitted to the observed PSDs (Ingram \& Done 2012; Ingram \& van der Klis 2013), but they tend to have somewhat steeper slope than the observed one in the frequency range $0.1-1 \mathrm{~Hz}$, as the data seems to be more consistent with two discrete peaks rather than the flat noise produced in this model (Belloni et al. 2002; Pottschmidt et al. 2003; Axelsson et al. 2005; Grinberg et al. 2014). Many studies suggest that the $f^{-1}$ power-law is a very crude approximation to the variability spectrum, as the PSD often shows a number of distinct peaks (the peaked noise components), which are usually fitted with Lorentzians (Nowak et al. 1999; Revnivtsev et al. 2000; Homan et al. 2001; Belloni et al. 2002, 2006). A possi- 


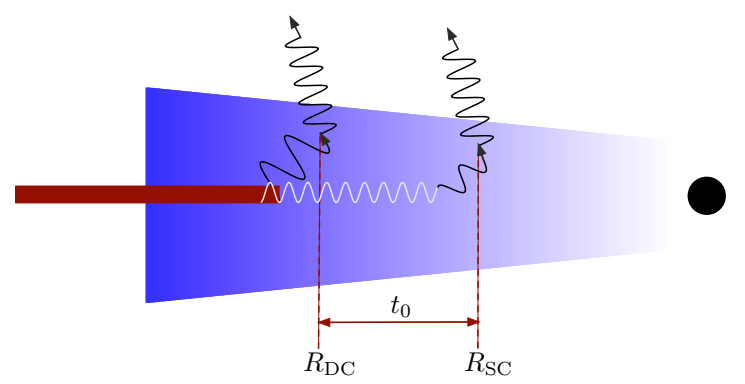

Figure 1. Sketch of the proposed geometry. The horizontal oscillations (white) show the propagating fluctuations of mass accretion rate, which are eventually transformed to radiation (black lines) that is up-scattered to Xrays through disk Comptonization (at radius $R_{\mathrm{DC}}$ ) and synchrotron Comptonization (at radius $R_{\mathrm{SC}}$ ). The light-curves produced by these two mechanisms should be similar, apart from damping of high frequencies in the disk Comptonization term and possible anti-correlation between them. There is a time delay between them, corresponding to the characteristic time required for the fluctuations to propagate from $R_{\mathrm{DC}}$ to $R_{\mathrm{SC}}$.

ble interpretation is that certain radii of the accretion flow are somewhat more variable than others. For instance, the enhancement of variability is expected in the transitional region between the cold disc and hot flow (Vikhlinin et al. 1994; Churazov et al. 2001), or at the hot flow inner boundary if the orbital and black hole spins are misaligned (Fragile 2009; Ingram \& Done 2012). Admittedly, more than forty years after the first publication of X-ray PSD from Cyg X-1 (Terrell 1972), no physical model was proposed to explain the complex multi-peak structure.

In this work we propose the first physical explanation for the peaked noise in the PSD of black hole binaries. We suggest that it is produced by the interference of two X-ray components, one being the disk Comptonization and the other being synchrotron Comptonization. Both components are variable as a result of propagating mass accretion rate fluctuations, both having the same PSD except for the possible damping at some frequency. An essential requirement is that there is a time delay between them, corresponding to the propagation time between the disk Comptonization radius, likely close to the cold disk truncation radius, and the radius where the synchrotron is produced and Comptonized within the hot accretion flow.

Such a scenario is supported by the spectral data. The cold disk is commonly believed to be the source of seed photons in the soft state (Done et al. 2007), but a number of arguments suggest alternative sources of seed photons in the hard state, such as synchrotron emission self-generated in the flow (Esin et al. 1997; Poutanen \& Vurm 2009; Malzac \& Belmont 2009; Veledina et al. 2013b; Poutanen \& Veledina 2014; Yuan \& Narayan 2014). It is then likely that we see contributions of both sources in the intermediate state.

In this paper we discuss dependence of resulting PSD shape on the model parameters and show that many observable features can be understood in terms of this mechanism. We apply the developed model to the multipeak PSDs observed in black hole binaries GX 339-4 and XTE J1848-288. We show that the delay time can be easily recovered from the PSD shape, with the help of which we can put constraints on the combination of disk height-to-radius ratio and viscosity parameter $\alpha(H / R)^{2}$, which is not measured directly for the hot flow.

\section{MODEL}

We consider a scenario where the disk is truncated at some radius and the hot flow exists within this truncation radius (Poutanen et al. 1997; Esin et al. 1997). In this geometry, the mass accretion rate fluctuations are excited at the cold accretion disc and are propagated from its inner boundary into the hot flow. The excited fluctuations lead to variations in the disc seed photons on the dynamical timescales at the truncation radius. The disc seed photons penetrate into the hot flow, where they are being Compton up-scattered to the X-rays. In addition to this, fluctuations in mass accretion rate $\dot{m}$ propagate from the disk truncation radius into the hot flow, exciting the $\mathrm{X}$-ray fluctuations again, this time via synchrotron Comptonization. The sketch of the considered geometry is shown in Figure 1.

Disc photons are effectively Comptonized in the region close to the truncation radius, however, not many disc photons penetrate to the synchrotron Comptonization region due to a small dilution factor. On the other hand, the dilution factor for synchrotron photons is not so small and they presumably may travel within the flow. However, the optical depth of the flow is of the order of unity (as commonly suggested by the spectral fitting, Zdziarski \& Gierliński 2004), so the emitted synchrotron photons are also likely to be Comptonized locally.

We assume that the observed fluxes produced by two mechanisms (Comptonization of the disk and the synchrotron photons) are directly proportional to the mass accretion rate and are additive, so the total X-ray lightcurve can be described by

$$
x(t)=\left[\varepsilon_{\mathrm{ds}} \dot{m}\left(t+t_{0}\right) * h(t)+\dot{m}(t)\right] / \sqrt{1+\varepsilon_{\mathrm{ds}}^{2}},
$$

where $\dot{m}$ denotes the light-curve of mass accretion rate fluctuations (throughout the paper, we use small letters for quantities in time domain and capital letters for frequency domain). The first term of this equation describes the emission coming from disk Comptonization, while the second term corresponds to the 

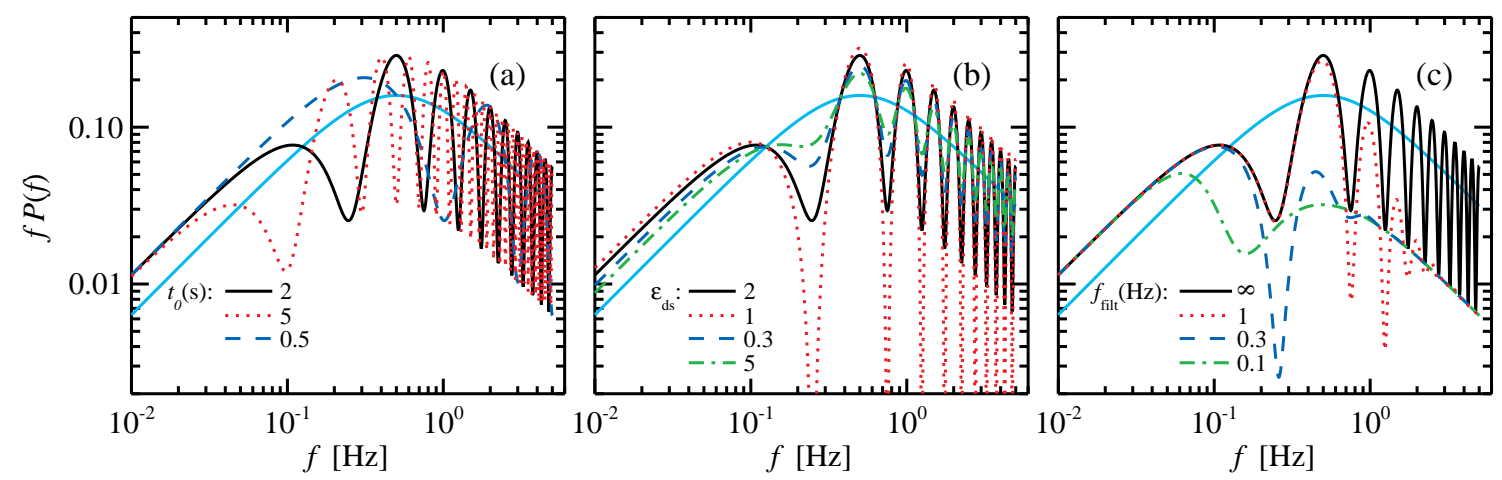

Figure 2. Power spectra obtained from the model. Black solid line in every panel corresponds to the fiducial parameter set $\left(t_{0}=2 \mathrm{~s}, \varepsilon_{\mathrm{ds}}=2\right.$, no damping). Cyan solid line in every panel is the seed Lorentzian, plotted for comparison purposes. Panel (a): dependence on the delay time $t_{0}$. Panel (b): dependence on $\varepsilon_{\mathrm{ds}}$. Panel (c): dependence on the damping frequency $f_{\text {filt }}$. Parameters for each curve can be found in Table 1.

synchrotron Comptonization. The parameter $\varepsilon_{\mathrm{ds}}$ regulates relative importance of the two terms. The denominator is the normalization factor. There is a time delay $t_{0}$ between the time disk photons Comptonize and the time the synchrotron photons Comptonize within the flow, corresponding to a characteristic propagation time from the truncation radius to the region where synchrotron Comptonization operates. This latter region might be extended along the radius, leading to some smearing of the variability from the synchrotron Comptonization light-curve compared to the mass accretion rate light-curve. However, the characteristic smearing time, of the order of viscous timescale at the synchrotron Comptonization radius, is much less that the delay time $t_{0}$, so the $\dot{m}(t)$ is still a good approximation for the synchrotron Comptonization light-curve. The disk Comptonization component is variable at the dynamical timescale of the truncation radius. It is lacking high-frequency signal, as it is originating at radii much farther than the characteristic radius of synchrotron Comptonization. This is modeled by a convolution $(*$ sign) of $\dot{m}(t)$ with the filter function $h(t)$. Its Fourier image is taken in the form

$$
H(f)=\frac{1}{\left(f / f_{\text {filt }}\right)^{4}+1},
$$

where $f_{\text {filt }}$ is the characteristic damping/filtering frequency.

The broadband X-ray PSD corresponding to the lightcurve from Equation (1) is described by

$$
|X|_{\mathrm{bb}}^{2}(f)=\frac{1+\varepsilon_{\mathrm{ds}}^{2} H^{2}(f)+2 \varepsilon_{\mathrm{ds}} H(f) \cos \left(2 \pi f t_{0}\right)}{1+\varepsilon_{\mathrm{ds}}^{2}}|\dot{M}|^{2}(f),
$$

where the cross-term proportional to $\cos \left(2 \pi f t_{0}\right)$ appears. This produces oscillations in the X-ray PSD and, depending on the value $\varepsilon_{\mathrm{ds}}$, can significantly reduce power at frequencies $f=(k-1 / 2) / t_{0}$, where $k$ is an integer number. The peaks appear at $f=k / t_{0}=$ $k f_{0}$. Hence, $f_{0}$ can be considered as fundamental fre-
Table 1. Parameters of numerical modelling for Figure 2.

\begin{tabular}{ccccc}
\hline \hline Panel & Line & $t_{0}(\mathrm{~s})$ & $\varepsilon_{\mathrm{ds}}$ & $f_{\text {filt }}(\mathrm{Hz})$ \\
\hline a,b,c & black solid & 2 & 2 & $\infty$ \\
a & red dotted & 5 & 2 & $\infty$ \\
a & blue dashed & 0.5 & 2 & $\infty$ \\
b & blue dashed & 2 & 0.3 & $\infty$ \\
b & red dotted & 2 & 1 & $\infty$ \\
b & green dot-dashed & 2 & 5 & $\infty$ \\
c & green dot-dashed & 2 & 2 & 0.1 \\
c & blue dashed & 2 & 2 & 0.3 \\
c & red dotted & 2 & 2 & 1.0 \\
\hline
\end{tabular}

quency and the other peaks as harmonics. The characteristic width of the peak set by the cosine term is $\delta f=2\left[(k+1 / 4) / t_{0}-k / t_{0}\right]=1 /\left(2 t_{0}\right)$, hence the peak quality factor $f / \delta f$ increases with increasing frequency. We note that the cross-term only redistributes the power between frequencies, the integral is preserved because the cosine dependence vanishes after integration.

\subsection{Dependence on parameters}

The study of the dependence of the PSD shape on model parameters can be done analytically using Equation (3). As an illustration, we use a single zero-centered Lorentzian function to describe the shape of the mass accretion rate $\mathrm{PSD}$

$$
|\dot{M}|^{2}(f)=\mathcal{L}(f) \equiv \frac{\Delta f / \pi}{(\Delta f)^{2}+f^{2}},
$$

where we take the characteristic width $\Delta f=0.5 \mathrm{~Hz}$. The resulting PSDs are shown in Figure 2 and the parameters for each curve are listed in Table 1 . The increase of time delay results in an increase of the number of humps, also the strength of the harmonic peaks relative to the low-frequency bump increases. A more pronounced cross-term (oscillations in PSD) appears when 


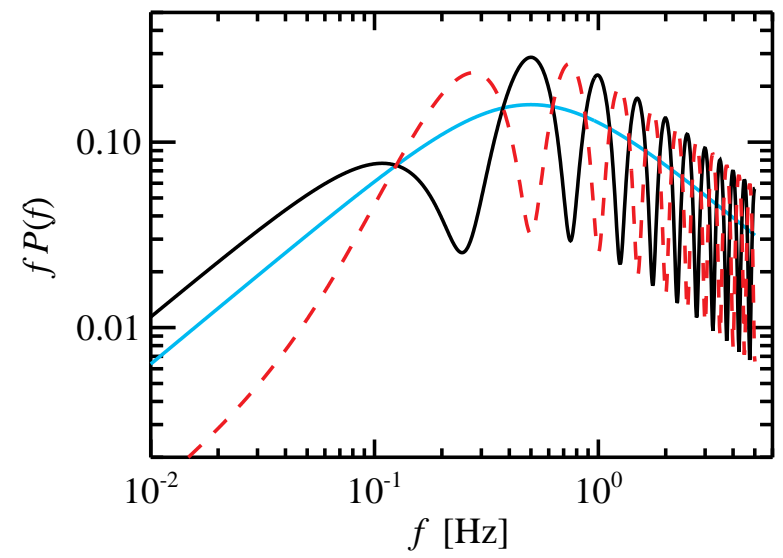

Figure 3. Power spectra obtained from the model for different signs of $\varepsilon_{\mathrm{ds}}$. Parameters for the black solid line can be found in Table 1 . The parameter $\varepsilon_{\mathrm{ds}}=-2$ for the red dashed line, other are the same as for the black line. Seed Lorentzian is shown with cyan solid line.

the parameter $\varepsilon_{\mathrm{ds}}$ approaches 1 , the oscillations disappear both at $\varepsilon_{\mathrm{ds}} \gg 1$ and $\varepsilon_{\mathrm{ds}} \ll 1$. The behavior is easily understood from Equation (3), suggesting that an interference between the two X-ray components is mostly seen when they have similar amplitudes and the effect vanishes once one of them dominates. This also implies that changing $\varepsilon_{\mathrm{ds}}$ to $1 / \varepsilon_{\mathrm{ds}}$ does not alter the PSD shape (here we assume no damping, $H(f)=1$ ), as the importance of the cosine term is determined by the ratio $2 \cos \left(2 \pi f t_{0}\right) /\left(\varepsilon_{\mathrm{ds}}+1 / \varepsilon_{\mathrm{ds}}\right)$. The dependence on damping frequency is rather straightforward: the smaller $f_{\text {filt }}$ is, the less the effects of the cross-term are and the less oscillations the PSD shows. Interestingly, for low filtering frequency, the PSD shows the double-hump shape, which resembles those often observed (e.g., Belloni et al. 2002).

\subsection{Different dependence on $\dot{m}$}

So far we assumed that variations of both disk and synchrotron Comptonization correlate with mass accretion rate fluctuations, however, this might not be realized for X-rays in some energy range. For instance, the disk Comptonization can produce spectral pivoting, as the amount of photons at lower energies are correlated with $\dot{m}$, while the amount of photons at higher energies is anti-correlated due to a decrease of the electron temperature (as reported, e.g., in Zdziarski et al. 2004). The variations of $\dot{m}$ may also cause spectral pivoting in the synchrotron Comptonization (Veledina et al. 2011a), light-curves of photons with energies above pivoting point are correlated with $\dot{m}$, but for photons with lower energies they are anti-correlated with $\dot{m}$. The behavior of these two components can be simply described as "softer when brighter" for the disc- and "harder when brighter" for synchrotron Comptonization. Depending on the position of the pivoting point and the energy

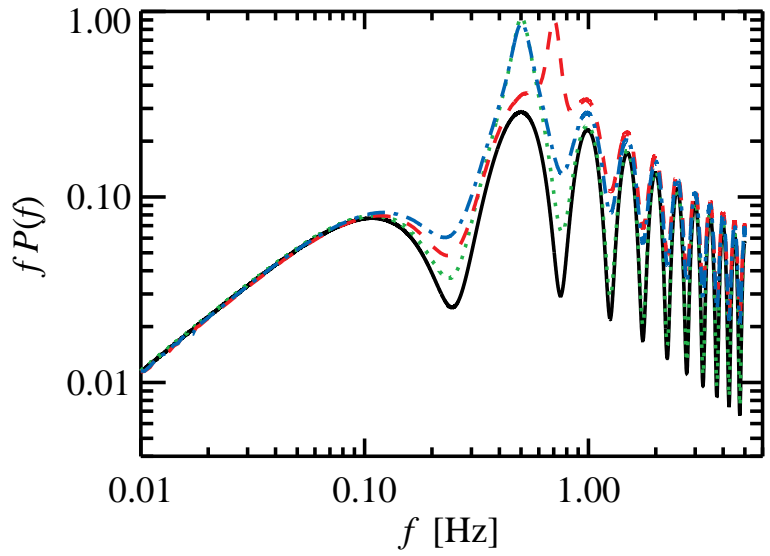

Figure 4. Model power spectra with additional contribution of the QPO. Parameters for black solid line can be found in Table 1. The QPO parameters are: $\Delta f_{\mathrm{QPO}}=0.05 \mathrm{~Hz}$, $\varepsilon_{1}=1, f_{\mathrm{QPO}}=0.7 \mathrm{~Hz}$ (red dashed line) and 0.5 (blue dotdashed line), other parameters are the same as for the black line. The PSD with the QPO coupled to the broadband variability in an additive way is shown with the green dotted line $\left(f_{\mathrm{QPO}}=0.5 \mathrm{~Hz}\right)$, we note the difference in oscillation amplitude in this case. The PSDs are renormalized to match at low frequencies.

range considered, both components can be seen correlated with accretion rate, anti-correlated with accretion rate (in these two cases they are correlated with each other) or one is correlated, the other is anti-correlated with $\dot{m}$ (in which case they are anti-correlated with each other).

Interestingly, two distinct variability patterns, with constant and changing spectral slope, were reported for Cyg X-1 (Zdziarski et al. 2002). Though found for the long-term variations, the behavior might be similar in the short-term variability, such as the "harder when brighter" pattern reported for GX 339-4 (Gandhi et al. 2008), and the "softer when brighter" behavior found in XTE J1118+480 (Malzac et al. 2003), suggesting Comptonization of synchrotron and disk to be dominant, respectively.

We investigate how the PSD changes if two sources are anti-correlated. This can be modeled by changing the sign of parameter $\varepsilon_{\mathrm{ds}}$. The resulting PSDs are shown in Figure 3. As can be easily shown analytically, the dips and peaks in PSD are now reversed. The peaks appear at frequencies $f=(k-1 / 2) / t_{0}$ and are related as odd numbers 1:3:5, while the dips are harmonically related and appear at $f=k / t_{0}$. The PSD shape below $\sim 0.05 \mathrm{~Hz}$ is different, now the PSD function is convex (its second derivative is positive), while for cases considered earlier it had a concave shape (negative second derivative).

\subsection{Coupling with $Q P O$}

Broadband variability in almost every X-ray PSD is accompanied by the low-frequency QPO. We assume 
that the QPO is coupled to the aperiodic variability in a multiplicative way, i.e. it modulates the accretion rate fluctuations. In this case, no simple analytical approximation can be obtained for the resulting PSD shape, so we proceed with the simulations of the X-ray lightcurves. The quantity $\dot{m}(t)$ is obtained from its PSD (zero-centered Lorentzian with $\Delta f=0.5 \mathrm{~Hz}$ as in previous cases) using the Timmer \& Koenig (1995) algorithm, with zero mean. The QPO PSD has a form of a narrow Lorentzian

$$
\mathcal{Q}_{n}(f)=\frac{\Delta f_{\mathrm{QPO}, n} / \pi}{\left(\Delta f_{\mathrm{QPO}, n}\right)^{2}+\left(f-n f_{\mathrm{QPO}}\right)^{2}},
$$

where subscript $n$ corresponds to a harmonic number. In this section, we consider only one harmonic. The QPO light-curves are obtained from this Lorentzian also using the Timmer \& Koenig (1995) algorithm, with zero mean.

We model the total light-curve as

$$
x(t)=\varepsilon_{\mathrm{ds}} \dot{m}_{\mathrm{filt}}\left(t+t_{0}\right)+[1+\dot{m}(t)]\left[1+\varepsilon_{1} q(t)\right]-1,
$$

where $q(t)$ is the QPO light-curve and constant $\varepsilon_{1}$ regulates its prominence (subscript 1 corresponds to the first harmonic). Such light-curve can arise, e.g., in the model where QPOs are produced by the rotating emission pattern of the hot flow (Veledina et al. 2013a), so the QPO modulates the mass accretion rate fluctuations in a multiplicative way. The most common scenario having the entire hot flow rotating is when it undergoes the Lense-Thirring precession (Fragile et al. 2007; Ingram et al. 2009).

As an illustration, we consider two limiting cases where the QPO central frequency coincides with the peak and with the dip of the PSD. The parameters are: $f_{\mathrm{QPO}}=0.7 \mathrm{~Hz}$ and $0.5 \mathrm{~Hz}$ (respectively); $\varepsilon_{1}=1$ and $\Delta f_{\mathrm{QPO}}=0.05 \mathrm{~Hz}$. We obtain the X-ray light-curves using Equation (6). To reduce the noise appearing due to coupling of QPO and broadband variability, we simulate 1000 light-curves, calculate their PSDs and average over the realizations. The resulting PSDs are shown in Figure 4. For a comparison, we also plot the model with the same parameters but without QPO (black line, see parameters in Table 1). We see that the presence of the QPO reduces the oscillation prominence at high frequencies.

The described model with the QPO requires numerical modelling, which may be computationally expensive when trying to fit the data directly. A simplest way to introduce the QPO into the model is just to add the QPO narrow Lorentzian to the model PSD. However, this procedure implicitly assumes that the QPO is related to the broadband variability in an additive way. We demonstrate the difference between multiplicative and additive QPO coupling in Figure 4. Increas- ing $\varepsilon_{\mathrm{ds}}$, i.e. suppressing the oscillations in the additive model, does not eliminate the difference between the two models: when the consistence is achieved at frequencies $f<f_{\mathrm{QPO}}$, the oscillations at frequencies $f>f_{\mathrm{QPO}}$ remain more pronounced in the additive model. Hence, the total PSD fitted using the analytical model and a QPO Lorentzian remains somewhat different from the one obtained using multiplicative model.

\section{IMPLICATIONS}

\subsection{Observational appearance}

Multiple peaks in the X-ray PSDs were reported, e.g., in GX 339-4 (Belloni et al. 2006; De Marco et al. 2015a), Cyg X-1 (Gilfanov et al. 1999), XTE J1748288 (Revnivtsev et al. 2000), SWIFT J1753.5-0127 (Hynes et al. 2009), XTE J1550-564 (Homan et al. 2001), GS 1124-68 (van der Klis 1995). The peaks can be fitted with a number of Lorentzians (zero-centered and QPO-like features), but the physical explanation behind this description is still missing. The same data can be explained in terms of the proposed model. We compute the PSD assuming the QPO is coupled to the broadband noise in an additive way, so the model PSD is given by

$$
|X|^{2}(f)=r\left[|X|_{\mathrm{bb}}^{2}+\varepsilon_{n} \mathcal{Q}_{n}(f)\right], n=1,2
$$

where the broadband variability $|X|_{\mathrm{bb}}^{2}$ is described by Equation (3) and the two QPO harmonics are described by narrow Lorentzians $\mathcal{Q}_{n}(f)$ (Equation 5). Parameters $\varepsilon_{n}$ describe relative importance of the QPO harmonics (first and second) and $r$ is the overall normalization. We take the mass accretion rate PSD in the form

$$
|\dot{M}|^{2}(f)=\frac{f^{\gamma_{1}}}{1+\left(f / f_{1}\right)^{\gamma_{2}}+\left(f / f_{2}\right)^{\gamma_{3}}}
$$

to mimic the double-broken power-law $\left(f_{1}\right.$ and $f_{2}$ are the two break frequencies). We expect the indices to be $\gamma_{1} \sim 0$ and $\gamma_{2} \sim 1$, as in the model of propagation fluctuations.

As the first example, we apply the developed model to the 2-15 keV range PSD of black hole binary GX 339-4 (see Figure 5). The data were taken during the hardintermediate state and reported in Belloni et al. (2006). The parameters of our modeling are listed in Table 2. We note that the delay and sign of $\varepsilon_{\mathrm{ds}}$ can be easily deduced from the characteristic frequencies of the peaks that appear at approximately 1.3, 4, 6, 9 and $13 \mathrm{~Hz}$. The third peak is likely affected by the QPO, however, even from this sequence the 1:3:5:7:9 ratio can be recovered. From this we deduce that the disk and synchrotron Comptonization light-curves are anti-correlated and the delay time is $t_{0}=0.38 \mathrm{~s}$, close to the model value.

Another example of model application is the hardintermediate state of black hole binary XTE J1748-288 


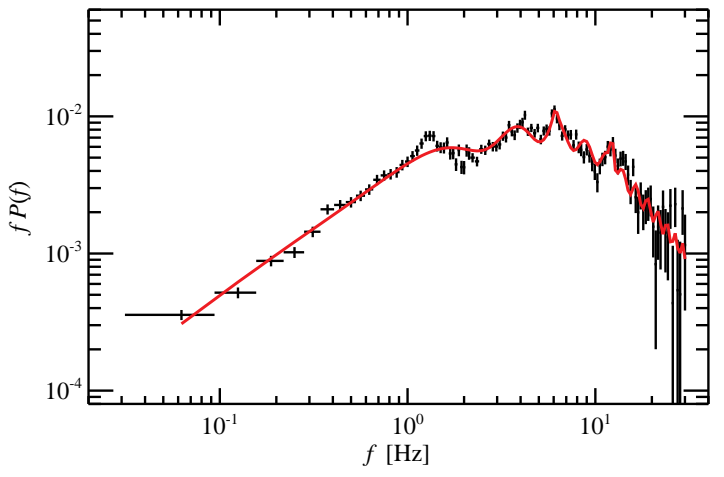

Figure 5. PSD simulated from the model (red solid line) resembles the observed in GX 339-4 (black crosses, Belloni et al. 2006). The model parameters are listed in Table 2 .

(Revnivtsev et al. 2000). We apply the same additive model and show the fit in Figure 6, parameters are listed in Table 2. Again, the $t_{0}$ value can be recovered from the peak positions.

Comparing the fitting parameters for these two objects we notice that the low-frequency break $f_{1}$ in the data on XTE J1748-288 is three times larger than in the data on GX 339-4, by the same factor as the ratio of the inferred delay times in these objects. This is in agreement with the picture that both the delay time and the low-frequency break of the PSD are related to the disk truncation radius. In addition, the disk fraction $\varepsilon_{\mathrm{ds}}$ is somewhat higher for XTE J1748-288, again consistent with the disk being closer to the $\mathrm{BH}$ in this object. The QPO frequency, yet another marker of the disk truncation radius, is higher in XTE J1748-288, again suggesting smaller truncation radius here.

When the disk is close to the precessing hot flow, its emission becomes modulated at the QPO frequency, so we have two sources of the QPO: one from the inner hot accretion flow, and the other from the modulation of the soft disk photons. Interference of these QPOs may potentially explain the type-B QPOs that also appear at the intermediate states. Interestingly, a PSD with a prominent type-B QPO and the PSD showed in Figure 5 were detected one hour apart (see Belloni et al. 2006).

\subsection{Viscous timescale marker}

The proposed model provides a potential to explore viscous timescale. The time-lags between two X-ray components can be attributed to the propagation time between two regions of Comptonization. The disk Comptonization likely occurs close to its truncation radius and the synchrotron Comptonization operates in the vicinity of the black hole. Hence, we can roughly estimate the propagation time with the viscous timescale at the truncation radius. The latter is determined as $1 / t_{\mathrm{visc}}=\Omega_{\mathrm{K}} \alpha(H / R)^{2}$, where $\Omega_{\mathrm{K}}$ is the Keplerian an-

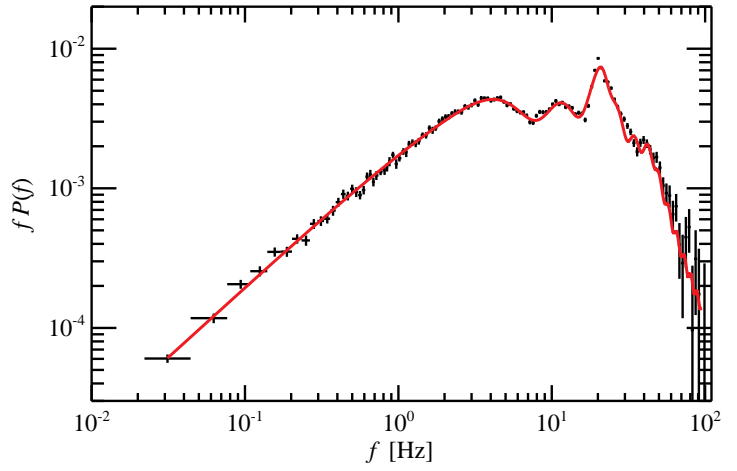

Figure 6. PSD simulated from the model (red solid line) resembles the observed in XTE J1748-288 (black crosses, Revnivtsev et al. 2000). The model parameters are listed in Table 2.

Table 2. Model parameters for Figures 5 and 6 .

\begin{tabular}{ccc}
\hline \hline Parameter & GX 339-4 & XTE J1748-288 \\
\hline$r$ & $6.78 \times 10^{-3}$ & $2.22 \times 10^{-3}$ \\
$f_{1}(\mathrm{~Hz})$ & 1.3 & 2.88 \\
$f_{2}(\mathrm{~Hz})$ & 5.0 & 20.1 \\
$\gamma_{1}$ & 0.077 & 0.007 \\
$\gamma_{2}$ & 0.96 & 1.45 \\
$\gamma_{3}$ & 3.14 & 5.11 \\
$\varepsilon_{\mathrm{ds}}$ & -0.068 & -0.086 \\
$t_{0}(\mathrm{~s})$ & 0.39 & 0.13 \\
$f_{\text {filt }}(\mathrm{Hz})$ & $\infty$ & $\infty$ \\
$f_{\mathrm{QPO}}(\mathrm{Hz})$ & 6.1 & 21 \\
$\Delta f_{\mathrm{QPO}, 1}(\mathrm{~Hz})$ & 0.34 & 3.0 \\
$\varepsilon_{1}$ & 0.08 & 1.0 \\
$\Delta f_{\mathrm{QPO}, 2}(\mathrm{~Hz})$ & 0.32 & 7.2 \\
$\varepsilon_{2}$ & 0.039 & 0.14 \\
\hline
\end{tabular}

gular velocity, $\alpha$ is the viscosity parameter and $H / R$ is the disk height-to-radius ratio. Scaling the radii to $50 R_{\mathrm{S}}\left(R_{\mathrm{S}}\right.$ is the Schwarzschild radius), the typical truncation radius in the hard state (Gilfanov et al. 2000; Basak \& Zdziarski 2016), we obtain

$$
\alpha\left(\frac{H}{R}\right)^{2}=\frac{0.05}{t_{\mathrm{visc}}}\left(\frac{M_{\mathrm{BH}}}{10 M_{\odot}}\right)\left(\frac{R}{50 R_{\mathrm{S}}}\right)^{3 / 2},
$$

where $M_{\mathrm{BH}}$ is the black hole mass. The disk truncation radius during the intermediate state is likely smaller. It cannot be very large, as it should contribute substantial amount of soft photons for Comptonization, but cannot be too small either, as it would produce too soft spectrum.

We impose constraints on $\alpha$ and $H / R$ by considering the range of possible truncation radii, $10-50 R_{\mathrm{S}}$, for $H / R<1$, and the condition of appearance of the 
QPO (from the solid-body precession of the hot disc, Fragile et al. 2007) $\alpha<H / R$. These limitations lead to the ranges $0.23<H / R$ and $0.01<\alpha<0.5$ for GX 339-4 and $0.33<H / R$ and $0.03<\alpha<0.73$ for XTE J1748288. Constraints on $H / R$ suggest that the propagation occurs in the geometrically thick flow in both cases. This implies that we are probing here the viscous parameter of the hot accretion flow, not that of the standard Shakura \& Sunyaev (1973) accretion disc, which is measured in cataclysmic variables, $\alpha_{\mathrm{CV}}=0.2$ (Smak 1999). The viscosity parameter of an advection dominated accretion flow is related to the maximal luminosity at which it can exist as $L_{\max } \sim 0.4 \alpha^{2} L_{\text {Edd }}$ (where $L_{\text {Edd }}$ is the Eddington luminosity), whereas for the luminous hot accretion flow, where the electron cooling is significant, the maximal luminosity is $\sim \alpha^{2} L_{\text {Edd }}$ (Yuan \& Narayan 2014). The latter hot flow model produces spectra resembling those of the hard-state black holes and Seyfert galaxies (Yuan \& Zdziarski 2004; Yuan et al. 2007). For $\alpha=0.5$ we obtain $L_{\max }=0.1 L_{\mathrm{Edd}}$ and $0.25 L_{\mathrm{Edd}}$ for advection-dominated and luminous hot accretion flow, respectively.

\subsection{Agreement with other observables}

The proposed model assumes that the X-rays are produced by two processes, the disk and synchrotron Comptonization, which are drastically different in terms of spectral behavior. We hence should be able to detect distinctive signatures of those in the spectral data. Investigations of the dependence of the X-ray spectral slope on luminosity show that at high luminosity the spectral index correlates with $L / L_{\mathrm{Edd}}$, but when this ratio falls below $\sim 0.01$, the dependence is reversed, and for lower $L / L_{\mathrm{Edd}}$ the index is larger (Sobolewska et al. 2011; Yang et al. 2015). The behavior is naturally explained in terms of change of seed photon source, the disk being at high $L / L_{\mathrm{Edd}}$ and the synchrotron contributing at small $L / L_{\mathrm{Edd}}$ (figure $7 \mathrm{~b}$ of Veledina et al. 2011b). Recently, an origin of seed photons for Comptonization was investigated using the data on the outburst decline of black hole binary SWIFT J1753.5-0127 (Kajava et al. 2016). The dependence of spectral index on the ratio of seed to Comptonized luminosity followed two different tracks at the peak and at the tail of the outburst. This was interpreted as the change of seed photon source, from the cold disk at the outburst peak to the synchrotron emission at the outburst tail. There should exist an overlap, where both mechanisms effectively operate, which likely corresponds to an intermediate state. Interestingly, this object was observed simultaneously in the X-rays and optical during the decline phase (Hynes et al. 2009). The X-ray PSD demonstrates the peaked noise component, and the optical/Xray cross-correlation function shows multiple dips and peaks shape, which can only be explained by considering two X-ray components (Veledina et al. in prep.).

In terms of the proposed model, the intermediate state corresponds to $\varepsilon_{\mathrm{ds}} \sim 1$, the hard state can be described by $\varepsilon_{\mathrm{ds}} \ll 1$ and the soft state, when there still exists a hot medium above the disc, can be described by $\varepsilon_{\mathrm{ds}} \gg 1$. The cross-term becomes more pronounced when the contributions of two terms are equal, thus in the low-luminosity hard state and very close to the soft state we do not expect multiple peaks. This general expectation is in agreement with the patterns seen in Cyg X-1 (Grinberg et al. 2014): the two-peak structure is most prominent at the intermediate spectral states.

We note that the cross-term only redistributes the overall power, but does not lead to a considerable suppression/enhancement of the root mean square (rms) variability amplitude, this can be seen by integrating Equation (3) over all frequencies. This implies that the presence of an additional component does not alter the rms-flux relation (Uttley \& McHardy 2001), which is an essential piece of evidence in favor of propagating accretion fluctuations model.

Gierliński et al. (2008) found that the high-frequency part of PSD, above the high-frequency break (if we utilize the double-broken power-law model), remains remarkably similar in a number of black holes. The slope and normalization constant are the same, in any particular object, during the state transition. They attributed this high-frequency part to a signature of the last stable orbit. We note that in our model, the slope of highfrequency part is not altered under change of any parameter. Resulting PSD at high $f$ has the same average slope as the seed Lorentzian has (apart from possible oscillations around the mean).

Recent investigations of variability at energies below $2 \mathrm{keV}$ with the $X M M$-Newton satellite suggest the variability is enhanced, compared to hard X-rays, at long timescales (Wilkinson \& Uttley 2009; Uttley et al. 2011; Cassatella et al. 2012). In the proposed model, probing different energy spectral ranges can be thought in terms of changing $\varepsilon_{\mathrm{ds}}$ parameter. Slight increase of power at low frequencies $(\sim 0.1 \mathrm{~Hz}$ in the present setup, but this depends on the seed Lorentzian parameters) can be obtained if relative contribution of disk Comptonization $\varepsilon_{\mathrm{ds}}=1$ for energies below $2 \mathrm{keV}$ and $\varepsilon_{\mathrm{ds}}=0.3$ for energies above $2 \mathrm{keV}$ (Figure 2b, red and blue lines, respectively). There also can be some difference in delay time, as the softer synchrotron Comptonization spectra are produced further away from the $\mathrm{BH}$, i.e. closer to truncation radius (Veledina et al. 2013b). Thus, for soft $\mathrm{X}$-rays the delay is less than for hard X-rays, hence from Figure 2a we deduce that the soft X-rays have larger variability amplitude at frequencies $\sim 0.01 \mathrm{~Hz}$ than hard X-rays. 
Another intriguing behavior is demonstrated by the time-lag spectra, which show the broken power-law dependence on energy in the low-frequency range: a weak increase of time-lag with energy above $\sim 2 \mathrm{keV}$ is opposed to the strong dependence on energy below this energy (Wilkinson \& Uttley 2009; Uttley et al. 2011). In addition, the dependence of time-lags on Fourier frequency depends on the energy range: it is a simple power-law for hard-medium energies, but the mediumsoft time-lags demonstrate a steeper slope at high frequencies, and the overall shape is a broken power-law. These features can be reproduced if we consider two components, one dominating in the soft energy range and the other is dominating at higher energies, which is delayed with respect to the soft one. We note that in this case the time-lag corresponds to viscous timescale, not to the light-travel time. Rapid increase of the time-lags at softer energies suggests a steep soft-component spectrum, it is not clear whether the disk Comptonization spectrum can account for that if we assume it is variable with constant spectral shape. Spectral evolution might play an important role here, e.g. it was shown that spectral pivoting can produce the broken power-law dependence of time-lags (Poutanen \& Fabian 1999).

Recently, two distinct classes of time-lags between the soft (presumably coming from the disc) and the hard (coming from the innermost parts of accretion flow) $\mathrm{X}$ rays were identified (De Marco et al. 2015b). The lags measured at low Fourier frequencies are of the order of $0.1 \mathrm{~s}$ and are likely connected to the propagation times from the cold disc to the Comptonization region. The measured time-lags are in agreement with the delays $\left(t_{0}\right)$ obtained in this paper. The reverberation lags constitute another class, in which case the soft band is delayed with respect to the hard band by about $10^{-3}-10^{-2} \mathrm{~s}$. Such echoes appear when the X-rays produced intrinsically within the flow are reflected from the cold accretion disc (see, e.g. Gilfanov et al. 2000; Poutanen 2002), and the X-ray PSD in this case is expected to have similar bumps due to the interference of the primary and reflected emission (Papadakis et al. 2016). However, due to substantial smearing of the reflected component, the bumps are likely less prominent than those proposed in this work.

An independent way to measure the viscosity parameter is from the low-frequency break of the X-ray power spectrum. The characteristic increase of the break frequency observed at the hard-to-soft state transition is likely caused by the decrease of the disc truncation radius (Gilfanov et al. 1999). Assuming the break frequency corresponds to the viscous timescale at the disc truncation radius, the viscosity and the disc aspect ratio $H / R$ can be recovered. Evolution of the $H / R$ throughout the outburst of BH binary XTE J1550-564 was traced by fitting to the low-frequency break of the $\mathrm{X}$ ray PSD (Ingram \& Done 2012). Such modeling relies on the prescribed distribution for the hot flow surface density distribution, so it would be interesting to compare the $H / R$ obtained by the two methods.

The proposed model can also be applied to other accreting compact objects, such as neutron star X-ray binaries and active galactic nuclei. Neutron star binaries reveal bumps (peaked noise) in their spectra (e.g., Hasinger \& van der Klis 1989; Belloni \& Hasinger 1990a; van der Klis 2006). They have an additional Xray component, the boundary layer, which can also interfere with the two considered components. Measuring the PSDs in active galactic nuclei is difficult due to an intermittent sampling of the frequency range of interest (Uttley \& McHardy 2005). The available data is generally consistent with a power-law or a broken power-law (Edelson \& Nandra 1999; Uttley et al. 2002), however, humps are sometimes observed (most prominent are in PKS 1346+26, some are seen in NGC 6860, a dip is present in 3C59, González-Martín \& Vaughan 2012; see also Emmanoulopoulos et al. 2016). Variability in active galactic nuclei can also studied using the structure function, which is expected to demonstrate a dip at the delay time.

\section{CONCLUSIONS}

Power spectra of accreting black hole binaries only roughly resemble flat-top noise (double-broken powerlaw). Instead, the data are more consistent with being produced by two or more components, usually fitted with Lorentzians. In some cases, up to six Lorentzians were required to explain its complex shape. We propose a physical scenario explaining appearance of such peaked noise in power spectra of accreting black hole binaries.

We consider the X-rays arising from two Comptonization processes: in up-scattering of the disk and the synchrotron photons. The two components are assumed to linearly respond to the mass accretion rate fluctuations, however, there is a time delay between them, corresponding to the propagation timescale between the two radii where Comptonization (of disk and of synchrotron photons) occurs. The total X-ray light-curve is obtained as a sum of two, time-shifted, mass accretion rate light-curves, and the power spectral density of such process constitutes a number of humps, appearing from the cross-term. We show that the humps central frequencies are harmonically related and the dips of power spectra follow 1:3:5 relation if the disk and synchrotron terms are correlated. If the two processes are anti-correlated, then the peaks follow 1:3:5 relation and the dips are harmonically related. We also show that coupling with the QPO reduces the cross-term ampli- 
tude, so the humps in the power spectra are less pronounced.

We discuss the consistency of this scenario with a number of observables such as: correlation of appearance of spectral bumps with the spectral properties, common shape of the power spectra at high frequencies (above the high-frequency break in terms of doublebroken power-law model), difference in power observed for soft and hard energies. We directly compare the model to the power spectra of black hole binaries GX 339-4 and XTE J1748-288 demonstrating numerous peaks. From the inferred time delay we put constraints on a combination of the viscosity parameter and the disk height-to-radius ratio $\alpha(H / R)^{2}$ and deduce that the propagation occurs in geometrically thick flow, $H / R>0.2$, and $\alpha<0.7$ for this hot flow. The model can be extended to describe power spectra of neutron stars and active galactic nuclei.

\section{ACKNOWLEDGEMENTS}

The author is grateful to Juri Poutanen and Andrzej Zdziarski for discussions and comments on the manuscript and Mike Revnivtsev and Tomaso Belloni for the data. I thank the referee for the suggestions which improved the paper. The work was supported by the Academy of Finland grant 268740.

\section{REFERENCES}

Arévalo, P., \& Uttley, P. 2006, MNRAS, 367, 801

Axelsson, M., Borgonovo, L., \& Larsson, S. 2005, A\&A, 438, 999

Basak, R., \& Zdziarski, A. A. 2016, MNRAS, 458, 2199

Belloni, T., \& Hasinger, G. 1990a, A\&A, 230, 103

-. 1990b, A\&A, 227, L33

Belloni, T., Psaltis, D., \& van der Klis, M. 2002, ApJ, 572, 392

Belloni, T., Parolin, I., Del Santo, M., et al. 2006, MNRAS, 367, 1113

Casella, P., Belloni, T., \& Stella, L. 2005, ApJ, 629, 403

Cassatella, P., Uttley, P., \& Maccarone, T. J. 2012, MNRAS, 427,2985

Churazov, E., Gilfanov, M., \& Revnivtsev, M. 2001, MNRAS, 321,759

De Marco, B., Ponti, G., Muñoz-Darias, T., \& Nandra, K. 2015a, MNRAS, 454, 2360

—. 2015b, ApJ, 814, 50

Done, C., Gierliński, M., \& Kubota, A. 2007, A\&A Rv, 15, 1

Edelson, R., \& Nandra, K. 1999, ApJ, 514, 682

Emmanoulopoulos, D., Papadakis, I. E., Epitropakis, A., et al. 2016, MNRAS, 461, 1642

Esin, A. A., McClintock, J. E., \& Narayan, R. 1997, ApJ, 489, 865

Fragile, P. C. 2009, ApJL, 706, L246

Fragile, P. C., Blaes, O. M., Anninos, P., \& Salmonson, J. D. 2007, ApJ, 668, 417

Gandhi, P., Makishima, K., Durant, M., et al. 2008, MNRAS, 390, L29

Gierliński, M., Nikołajuk, M., \& Czerny, B. 2008, MNRAS, 383, 741

Gilfanov, M., Churazov, E., \& Revnivtsev, M. 1999, A\&A, 352, 182

—. 2000, MNRAS, 316, 923

González-Martín, O., \& Vaughan, S. 2012, A\&A, 544, A80

Grinberg, V., Pottschmidt, K., Böck, M., et al. 2014, A\&A, 565, A1

Hasinger, G., \& van der Klis, M. 1989, A\&A, 225, 79

Hogg, J. D., \& Reynolds, C. 2015, ArXiv e-prints, arXiv: 1512.05350

Homan, J., Wijnands, R., van der Klis, M., et al. 2001, ApJS, 132,377

Hynes, R. I., O’Brien, K., Mullally, F., \& Ashcraft, T. 2009, MNRAS, 399, 281

Ingram, A., \& Done, C. 2011, MNRAS, 415, 2323

-. 2012, MNRAS, 419, 2369

Ingram, A., Done, C., \& Fragile, P. C. 2009, MNRAS, 397, L101

Ingram, A., \& van der Klis, M. 2013, MNRAS, 434, 1476
Kajava, J. J. E., Veledina, A., Tsygankov, S., \& Neustroev, V. 2016, ArXiv e-prints, arXiv:1603.08796

Kotov, O., Churazov, E., \& Gilfanov, M. 2001, MNRAS, 327, 799

Lyubarskii, Y. E. 1997, MNRAS, 292, 679

Malzac, J., Belloni, T., Spruit, H. C., \& Kanbach, G. 2003, A\&A, 407, 335

Malzac, J., \& Belmont, R. 2009, MNRAS, 392, 570

Nolan, P. L., Gruber, D. E., Matteson, J. L., et al. 1981, ApJ, 246, 494

Nowak, M. A., Wilms, J., \& Dove, J. B. 1999, ApJ, 517, 355

Papadakis, I., Pecháček, T., Dovčiak, M., et al. 2016, A\&A, 588, A13

Pottschmidt, K., Wilms, J., Nowak, M. A., et al. 2003, A\&A, 407, 1039

Poutanen, J. 2002, MNRAS, 332, 257

Poutanen, J., \& Fabian, A. C. 1999, MNRAS, 306, L31

Poutanen, J., Krolik, J. H., \& Ryde, F. 1997, MNRAS, 292, L21

Poutanen, J., \& Veledina, A. 2014, SSRv, 183, 61

Poutanen, J., \& Vurm, I. 2009, ApJL, 690, L97

Revnivtsev, M. G., Trudolyubov, S. P., \& Borozdin, K. N. 2000, MNRAS, 312, 151

Shakura, N. I., \& Sunyaev, R. A. 1973, A\&A, 24, 337

Smak, J. 1999, AcA, 49, 391

Sobolewska, M. A., Papadakis, I. E., Done, C., \& Malzac, J. 2011, MNRAS, 417, 280

Terrell, Jr., N. J. 1972, ApJL, 174, L35

Timmer, J., \& Koenig, M. 1995, A\&A, 300, 707

Uttley, P., \& McHardy, I. M. 2001, MNRAS, 323, L26

-. 2005, MNRAS, 363, 586

Uttley, P., McHardy, I. M., \& Papadakis, I. E. 2002, MNRAS, 332,231

Uttley, P., Wilkinson, T., Cassatella, P., et al. 2011, MNRAS, 414, L60

van der Klis, M. 1995, in Frontier Objects in Astrophysics and Particle Physics, ed. F. Giovannelli \& G. Mannocchi, 213

van der Klis, M. 2006, in Compact stellar X-ray sources, Cambridge Astrophysics Series, No. 39, ed. W. Lewin \& M. van der Klis (Cambridge: Cambridge University Press), 39-112

Veledina, A., Poutanen, J., \& Ingram, A. 2013a, ApJ, 778, 165 Veledina, A., Poutanen, J., \& Vurm, I. 2011a, ApJL, 737, L17 —. 2013b, MNRAS, 430, 3196

Veledina, A., Vurm, I., \& Poutanen, J. 2011b, MNRAS, 414, 3330

Vikhlinin, A., Churazov, E., \& Gilfanov, M. 1994, A\&A, 287, 73

Wilkinson, T., \& Uttley, P. 2009, MNRAS, 397, 666

Yang, Q.-X., Xie, F.-G., Yuan, F., et al. 2015, MNRAS, 447, 1692 
Yuan, F., \& Narayan, R. 2014, ARA\&A, 52, 529

Yuan, F., \& Zdziarski, A. A. 2004, MNRAS, 354, 953

Yuan, F., Zdziarski, A. A., Xue, Y., \& Wu, X.-B. 2007, ApJ, 659,541

Zdziarski, A. A., \& Gierliński, M. 2004, Progr. Theor. Phys. Suppl., 155, 99
Zdziarski, A. A., Gierliński, M., Mikołajewska, J., et al. 2004, MNRAS, 351, 791

Zdziarski, A. A., Kawabata, R., \& Mineshige, S. 2009, MNRAS, 399, 1633

Zdziarski, A. A., Poutanen, J., Paciesas, W. S., \& Wen, L. 2002, ApJ, 578, 357 